\title{
Caracterização elástica de compensados de madeira utilizados e reutilizados em fôrmas através de excitação por impulso
}

\author{
Elastic characterization of plywood used and \\ reused in forms through impulse excitation
}

\author{
Vinnicius Dordenoni Pizzol ${ }^{1}$, Judy Norka Rodo Mantilla ${ }^{2}$, \\ Edgar Vladimiro Mantilla Carrasco ${ }^{3}$
}

\author{
${ }^{1}$ Programa de Pós-graduação em Engenharia de Estruturas, UFMG, Av. Antônio Carlos, 6627 - Escola de Engenharia - \\ Bloco $1-4^{\circ}$ andar, sala 4215, Pampulha, Belo Horizonte - MG - CEP 31270-901 \\ e-mail: vpizzol@hotmail.com \\ ${ }^{2}$ Faculdade de Engenharia e Arquitetura, Universidade FUMEC, rua Cobre, 200, Cruzeiro, Belo Horizonte - MG - CEP \\ 30310-190, e-mail: judy.mantilla@fumec.br \\ ${ }^{3}$ Escola de Arquitetura, UFMG, Rua Paraíba, 697, Funcionários, Belo Horizonte - MG - CEP 30130-140 e-mail: mantil- \\ la@dees.ufmg.br
}

\section{RESUMO}

As fôrmas de madeira são os elementos responsáveis em dar a forma que se deseja ao concreto fresco e proporcionar proteção contra impactos e variações de temperatura no seu processo de cura. As chapas de madeira compensada são os elementos mais utilizados na construção civil nesse processo. $\mathrm{O}$ reuso das chapas é comum durante a obra, não ultrapassando 10 vezes. No entanto, o material poderá ter variação nas propriedades mecânicas. O primeiro objetivo deste trabalho foi determinar experimentalmente a variação do módulo de elasticidade em função da quantidade de reuso do compensado de madeira. O ensaio utilizado foi de flexão a três pontos, com o qual foi determinado o módulo de elasticidade. Durante uma obra, o envio de amostragens para análises laboratoriais requer tempo, entretanto a empresa construtora não pode esperar esse tempo para dar continuidade à execução da construção. Se as características do compensado pudessem ser conhecidas na obra, de forma rápida e prática, o serviço poderia ser feito com segurança e rapidez. O segundo objetivo foi apresentar uma técnica não destrutiva que se encaixa neste contexto a qual é a excitação por impulso. O delineamento experimental foi de três tratamentos e sete repetições por tratamento. As amostras utilizadas no ensaio de excitação por impulso e as do ensaio destrutivos foram as mesmas. Os resultados indicam que a umidade sofre pouca influência na reutilização das formas, já a densidade tem bastante variação devido à perda de material durante a reutilização. O módulo de elasticidade tem uma diminuição de $48 \%$ após a quinta reutilização. O ensaio não destrutivo utilizando excitação por impulso mostra-se adequado para estimar o módulo de elasticidade das formas de concreto.

Palavras-chave: modulo de elasticidade, ensaios não destrutivos, construção civil.

\section{ABSTRACT}

The wood forms are the responsible elements in giving the desired shape to the fresh concrete and to provide protection against impacts and temperature variations in its curing process. Plywood sheets are the elements most used in building for this process. The reuse of the plates is common during the work - not exceeding 10 times. However, the material may have variation in its mechanical properties. The primary goal of this work was to experimentally determine the modulus of elasticity as a function of the amount of reuse of the plywood. The test used was a three-point bending, in which the modulus of elasticity was determined. During a work, the sending of samples for laboratory analysis requires time. Nevertheless, the construction company cannot wait such time to continue the execution of the construction. If the characteristics of the plywood could be determined quickly and practically, the service could have been done safely and quickly. The second goal was to present a non-destructive technique which fits this context, that is, the impulse excitation. The experimental design took three treatments and seven replicates per each treatment. The same samples for the impulse excitation test and the destructive trials were used. The results show that the moisture has little influence on the reuse of the forms, since the density has enough variation due to the loss of material during reuse. The modulus of elasticity has a decrease of $48 \%$ after the fifth reuse. The non-destructive test using 
impulse excitation is suitable for estimating the modulus of elasticity of concrete forms.

Keywords: Modulus of elasticity, non-destructive tests, civil construction.

\section{INTRODUÇÃO}

O mercado da construção civil para a madeira é altamente promissor, por ser um material versátil que reduz o tempo de construção, favorece a qualidade final da obra e assegura uma construção seca e sustentável, dentro do conceito de desenvolvimento sustentável. É possível construir tudo com auxílio da madeira, inclusive edificações de múltiplos andares. No entanto, no Brasil o maior obstáculo é a falta de conhecimento de temas abrangentes sobre a madeira. Os profissionais da construção carecem de entender os valores técnicos da madeira e compreender a trabalhabilidade desse material. Cabe a ressalva que para a devida aplicação seja distinguida e popularizada, é necessária uma difusão do conhecimento [1].

A evolução tecnológica e o desenvolvimento de novos materiais derivados da madeira, de propriedades melhoradas, constituem também fortes fatores motivadores na aposta na construção com madeira em altura. Por um lado, os novos materiais derivados da madeira, com propriedades mecânicas melhoradas, possuem um processo de fabricação padronizado e rigoroso que garante a qualidade do material [2].

A produção de painéis de madeira foi uma grande evolução tecnológica e gerou grande crescimento na indústria com o passar dos anos, principalmente no ramo de construção civil. Devido a fragilidade da madeira maciça em vencer grandes vãos, quando submetido a um estado plano de tensões, a heterogeneidade do produto, a instabilidade dimensional e a carência do mercado nesse seguimento, teve que ser desenvolvido tecnologias capazes de suprir essa lacuna e assim, foi intensificado o desenvolvimento de chapas de compensado, chapas de partículas de madeira, madeira laminada colada e mais recente madeira laminada colada cruzada [3].

O compensado é um material versátil que pode ser utilizado, estruturalmente, de várias formas. Ele é feito a partir de lâminas de madeiras com fina espessura sobrepostas e unidas por um adesivo resistente, buscando uma equivalência das propriedades elásticas e de resistências nas direções mais significativas da chapa, largura e comprimento. A prensagem em alta pressão dessas lâminas garante uma boa resistência mecânica ao material. Outra vantagem é que o compensado pode ser reutilizado várias vezes, mas para isso é necessário que se conheça suas características [4].

Uma maneira de utilizar o compensado na construção civil é durante a obra em fôrmas, que são moldes usados para dar a forma que se deseja ao concreto fresco [5,6]. Segundo a NBR 15696 [7], as fôrmas são estruturas provisórias que servem para moldar o concreto fresco, resistindo a todas as ações provenientes das cargas variáveis resultantes das pressões do lançamento do concreto fresco, até que o concreto se torne autoportante. Além de proteger o concreto novo, limitar a perda de água, evitar a fuga de finos, permitir a obtenção de superfícies pré-determinadas, possibilitar o posicionamento de outros elementos como escoras, garantem a geometria da peça.

Normalmente as fôrmas de compensado são utilizadas mais de uma vez durante o período de uma obra, o que faz com que a madeira constantemente esteja em contato com água do concreto, que pode afetar diretamente nas suas propriedades físicas e mecânicas devido a sua higroscopicidade, além do enfraquecimento da ligação adesiva entre as lâminas. Outro ponto é o comprometimento das propriedades pelo uso de pregos e parafusos que, ao serem retirados, danificam a fôrma. Sendo necessária assim, uma investigação rápida e precisa sobre a redução das propriedades elásticas do material e da densidade.

Otani e Pereira [8] utilizaram a técnica para a caracterização de madeiras e derivados através de excitação por impulso, ensaio não destrutivo para a determinação dos módulos de elasticidade dinâmicos e do amortecimento através das frequências naturais de vibração. Técnicas não destrutivas referem-se à inspeção de materiais e equipamentos, onde incluem métodos capazes de determinar informações sobre produtos e características tecnológicas dos materiais sem danifica-los ou desperdiça-los, sendo rápidos e precisos, podendo assim validar a variação das propriedades elásticas da fôrma de compensado reutilizada.

Assim os objetivos deste trabalho são: - determinar a influência do reuso das formas na umidade, na densidade e no módulo de elasticidade do compensado de madeira, - determinar a eficiência do ensaio não destrutivo utilizando excitação por impulso para estimar o módulo de elasticidade do compensado de madeira.

\section{MATERIAIS E MÉTODOS}

Esta seção contém informações do material, dos equipamentos, dos ensaios experimentais que foram utilizados no trabalho e o princípio e teoria da técnica de excitação por impacto. Os ensaios foram realizados no Centro de Pesquisa Avançado da Madeira e Novos Materiais $\left(\mathrm{CPAM}^{3}\right)$, da Universidade Federal de Minas Ge- 
rais (UFMG).

\subsection{Material}

Foram adquiridas junto a empresas do ramo de construção civil no município de Belo Horizonte - MG, amostras de painéis compensados. A espécie de madeira utilizadas na produção dos compensados foi a Virola surinamensis (rol) Warb, da família Myristicaceae.

A região de ocorrência da Virola é em margens alagáveis dos rios e igapós, como também em várzeas, principalmente nos estados do Amazonas e do Pará. É citada como espécie de bom crescimento em plantios homogêneos. Tem como características: densidade baixa, cerne de coloração bege-claro-rosado e pouco distinto do alburno. Utilizada na confecção de compensados por ser uma madeira de fácil trabalhabilidade com ferramenta de corte resultando em uma superfície lisa e de fácil colagem, [13].

\subsection{Amostragem}

$\mathrm{O}$ delineamento experimental foi de três tratamentos e sete repetições por tratamento. $\mathrm{O}$ tipo de tratamento foi definido com a disponibilidade de amostras das empresas e do número de vezes de reuso (quantas vezes o compensado de madeira foi utilizado). Na Tabela 1, estão descritos os tratamentos e a quantidade de amostras por ensaio, cabendo salientar que as amostras utilizadas no ensaio de excitação por impulso e as do ensaio destrutivos foram as mesmas.

Tabela 1: Delineamento experimental.

\begin{tabular}{c|c|c|c}
\hline TRATAMENTO & DENSIDADE & UMIDADE & EXCITAÇÃO POR IMPULSO E ENSAIO DESTRTUTIVO \\
\hline Material novo & 7 amostras & 7 amostras & 7 amostras \\
\hline $1^{\text {a }}$ utilização & 7 amostras & 7 amostras & 7 amostras \\
\hline $5^{\text {a }}$ utilização & 7 amostras & 7 amostras & 7 amostras \\
\hline
\end{tabular}

\subsection{Densidade aparente}

As amostras para o ensaio de densidade aparente foram cortadas e ensaiadas de acordo a NBR 9485 [9]. Para a mensuração das dimensões utilizou-se um paquímetro digital, sendo realizadas três repetições para cada aferição e a massa em uma balança digital com precisão de $\pm 0,001$ g. A Equação 1 foi utilizada para calcular a densidade aparente de cada amostra e por seguinte calculou-se a média e o desvio padrão por tratamento.

$$
\rho_{a p .}=\frac{m}{\text { c.l.e }}
$$

Onde: pap.: densidade aparente (g/cm-3); m: massa (g); c: comprimento da amostra (cm); 1: largura da amostra $(\mathrm{cm})$ e; e: espessura da amostra $(\mathrm{cm})$.

\subsection{Teor de umidade}

O ensaio de umidade foi realizado segundo as diretrizes da NBR 9484 [10], onde inicialmente as amostras foram passadas em uma balança digital $( \pm 0,001 \mathrm{~g})$ determinando-se a massa inicial, em seguidas levadas a uma estufa a temperatura de $103^{\circ} \mathrm{C} \pm 2^{\circ} \mathrm{C}$, onde foram mantidas até que a massa aferida fosse constante e assim determinada a massa seca. Com a Equação 2 foi calculada a umidade de cada amostra e retirou-se a média de cada tratamento.

$$
U(\%)=\frac{m_{s}-m_{u}}{m_{s}} \cdot 100
$$

Onde: U (\%).: umidade (\%); ms: massa inicial (g) e; ms: massa seca (g).

\subsection{Ensaio de flexão estática}

A determinação do modulo de elasticidade foi feita de acordo com a NBR 9533 [11], sendo que o vão ensaiado deve ser 25 vezes a espessura da peça e o comprimento da amostra deve ser o tamanho do vão mais $5 \mathrm{~cm}$, como cada amostra tinha $2 \mathrm{~cm}$ de espessura, o vão utilizado foi de $50 \mathrm{~cm}$ e o comprimento da amostra $55 \mathrm{~cm}$. E a largura, pré-estabelecida pela norma, foi de $7,5 \mathrm{~cm}$. Cabendo salientar que as amostras utilizadas no ensaio de flexão e de excitação por impulso foram as mesmas, primeiramente, ensaiou-se no equipamento não 
destrutivo e depois no destrutivo.

O equipamento utilizado para o ensaio de flexão foi a maquina EMIC DL 3000 acoplado com um medidor de deslocamento EMIC no meio do vão, ligado ao sistema de aquisição de dados da máquina. $\mathrm{O}$ ensaio foi a flexão em três pontos, conforme Figura 1. Para o calculo do módulo de elasticidade de cada amostra foi utilizado a Equação 3:

$$
E=\frac{L^{3} \cdot\left(F_{2}-F_{1}\right)}{4 \cdot l \cdot e^{3} \cdot\left(s_{2}-s_{1}\right)}
$$

Onde: E: módulo de elasticidade (MPa); L: tamanho dos vãos (m); 1: largura da amostra (m); e: espessura da amostra (m); F2 - F1: incremento de carga $(\mathrm{N})$ e; s2 - s1: incremento de deflexão no ponto central do vão (m).

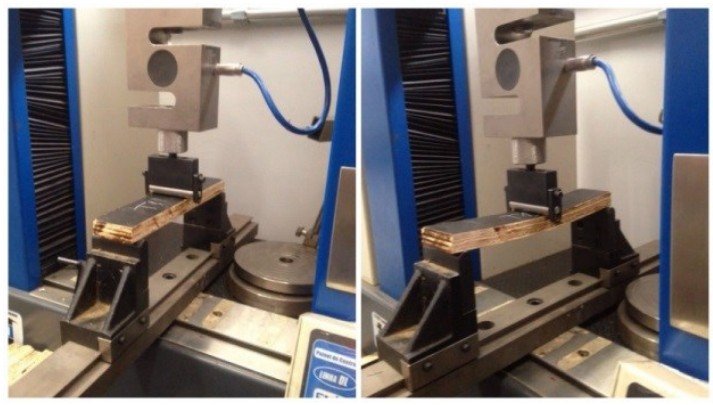

Figura 1: Ensaio e equipamento de flexão.

\subsection{Excitação por impulso}

A excitação por impulso é um ensaio não destrutivo para a determinação dos módulos de elasticidade dinâmicos e do amortecimento de materiais através das frequências naturais de vibração, a técnica utiliza a norma ASTM E1876 [12] como base em suas determinações e o princípio do aparelho Sonelastic (Figura 2).

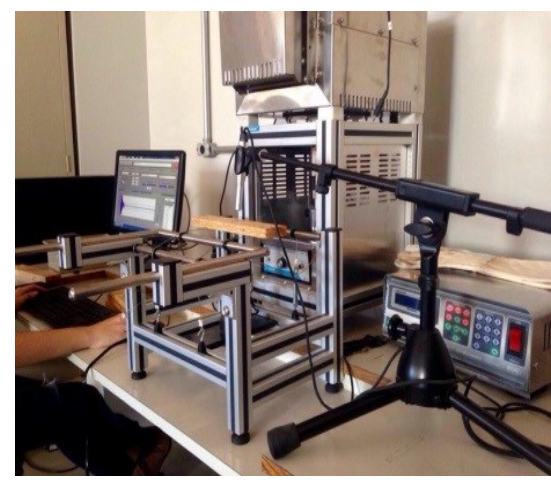

Figura 2: Ensaio e equipamento de excitação por impulso.

O princípio de funcionamento do equipamento Sonelastic $®$ baseia-se na excitação das frequências por meio de um pulsador, seguido da captação da resposta acústica por um sensor, Figura 2. Então aplica-se um tratamento matemático ao sinal (transformada de Fourier) para a obtenção do espectro de frequências correspondente e, a partir disso, é calculado o módulo elástico dinâmico [8]. Para isso é necessário fornecer a geometria, a massa e as dimensões das amostras previamente mensuradas por um paquímetro e uma balança digital e que juntamente com a frequência, obtida pelo equipamento, é possível determinar o módulo de elasticidade dinâmico através da Equação 4.

$$
\mathrm{Ed}=0,9465\left(\frac{m f_{f}^{2}}{l}\right)\left(\frac{c^{3}}{e^{3}}\right) T
$$

Em que: Ed: Módulo de elasticidade dinâmico (MPa); ff: frequência na flexão (Hz); m: massa (g); c: comprimento (m); 1: largura (m); e: espessura (m) e; T: coeficiente de correção. 


\section{RESULTADOS E DISCUSSÃO}

\subsection{Umidade e Densidade aparente}

De acordo com o ensaio de umidade, foi possível constatar que as amostras têm uma umidade de equilíbrio dentro do esperado para de Belo Horizonte, que variam entre 12 a 14\%, conforme Tabela 2. No entanto, as amostras já utilizadas apresentaram ganho de umidade devido ao contato com a água utilizada na concretagem.

Tabela 2: Resultados de umidade e densidade aparente.

\begin{tabular}{c|c|c}
\hline TRATAMENTO & UMIDADE $\mathbf{( \% )}$ & DENSIDADE $\mathbf{( g / \mathbf { c m } ^ { - 3 } )}$ \\
\hline Material novo & $12,89 \pm 0,02$ & $0,55 \pm 0,02$ \\
\hline $1^{\text {a }}$ utilização & $13,78 \pm 0,03$ & $0,51 \pm 0,01$ \\
\hline $5^{\text {a }}$ utilização & $13,98 \pm 0,02$ & $0,51 \pm 0,02$ \\
\hline
\end{tabular}

Já com o ensaio de densidade aparente, foi possível comprovar que as amostras perdem densidade após o uso, conforme mostrado na Tabela 2. As amostras sem uso apresentaram densidade de $0,55 \mathrm{~g} / \mathrm{cm}^{3} \mathrm{e}$, as reutilizadas, $0,51 \mathrm{~g} / \mathrm{cm}^{3}$. Foi possível observar que o material reutilizado continha danos, ocasionados por pregos e parafusos, e até mesmo por pedaços que ficaram presos no molde após a desforma, como se pode constatar na Figura 3. Isso acontece porque não foi utilizado desmoldante, produto disponível no mercado que facilita a retirada da placa de compensado em contato com o concreto. Os resultados das amostras sem uso estão semelhantes ao trabalho de Júnior e Garcia [13], com uma densidade aparente de 0,55 e teor de umidade de $12,1 \%$.

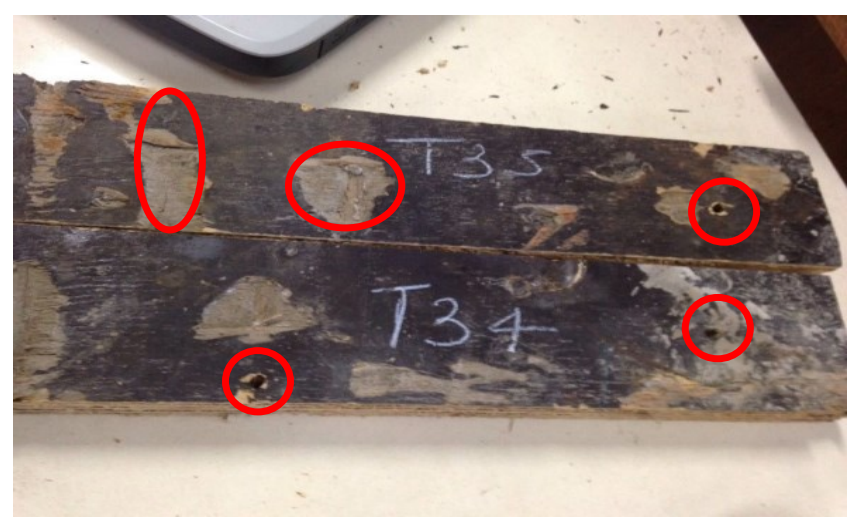

Figura 3: Furos e desfeitos nas amostras com 5 utilizações.

\subsection{Módulo de elasticidade da flexão}

A partir dos resultados obtidos com o ensaio de flexão estática, constatou-se que, com o uso do painel apenas uma vez, não houve a redução do módulo de elasticidade (E), e já no painel com mais vezes de reutilização, a redução do módulo foi de aproximadamente de $48 \%$, representado na Figura 4 . O valor do módulo para o material sem uso foi de 2871,83 MPa, para o usado uma vez foi de 2856,78 MPa e para a amostra usada 5 vezes foi de $1497,77 \mathrm{MPa}$. 


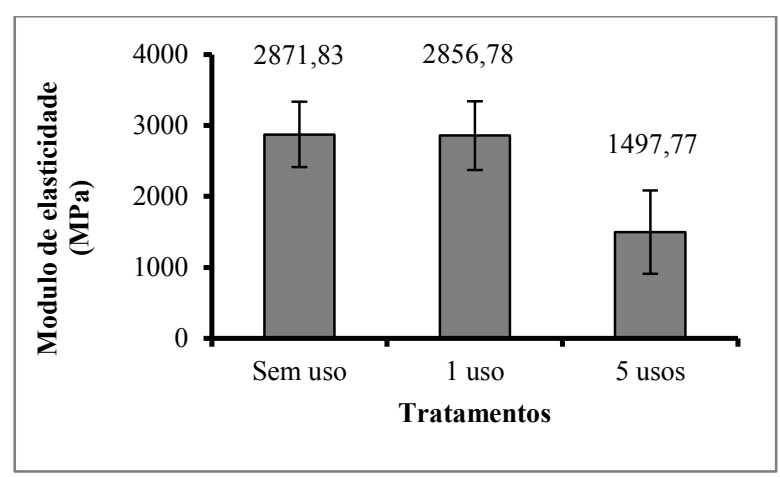

Figura 4: Gráfico Ensaio de Flexão

O valor mínimo encontrado no ensaio acima, 1497,77MPa, se aproximou do resultado de Stangerlin et al. [14] para condições de umidade semelhantes (12\%) em madeira de Carya illinoinensis, sendo o valor do módulo de elasticidade de 1626,19 MPa.

O reuso mostrou que há uma redução do módulo de elasticidade em quase $50 \%$, com isso a flecha que é inversamente proporcional irá dobrar, o que pode comprometer a eficácia do sistema de fôrma. Se essa questão não for considerada, a perda de elasticidade pode gerar grandes custos com compra de material em maior quantidade, além de tornar a estrutura instável. A importância de especificar um limite as flechas em fôrmas, tem a finalidade de evitar ondulações na superfície das estruturas, comprometendo sua aparência e sua resistência $[5,6,7]$.

\subsection{Estimativa do módulo de elasticidade através do ensaio de excitação por impulso}

Na Figura 5 estão os módulos de elasticidade dinâmicos obtidos através do ensaio não destrutivo no equipamento Sonelastic ${ }^{\circledR}$. Os resultados também mostraram que o material sem uso e utilizado uma vez mantém semelhanças com em relação ao módulo de elasticidade, já o que foi utilizado 5 vezes a redução é de aproximadamente $48 \%$, assim como encontrado na flexão estática (ensaio destrutivo).

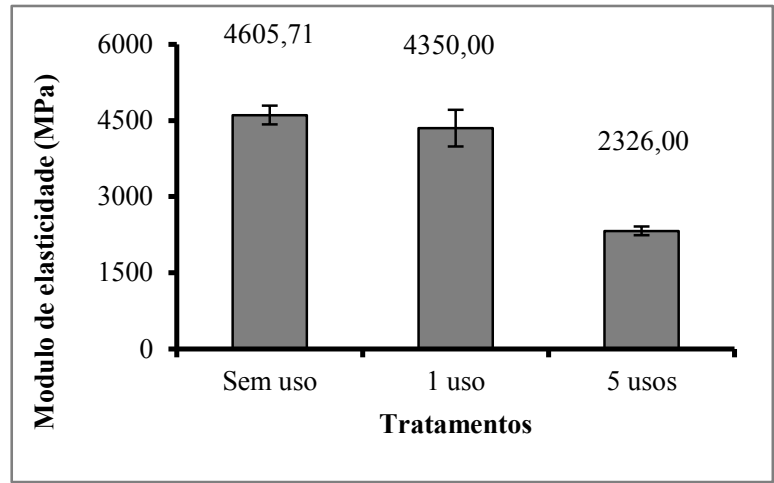

Figura 5: Gráfico Ensaio de excitação por impulso

\subsection{Correlação entre excitação por impulso e ensaio de flexão}

Conforme apresentado no gráfico da Figura 6 a correção entre o ensaio de flexão e de excitação por impulso é linear. Outro fator, é que o $\mathrm{R}^{2}$ (coeficiente de determinação) do Sonelastic apresentou de 0,73 , valor de correlação próximo as pesquisas com outros equipamentos não destrutivos, como ultrassom, utilizado no trabalho de Nogueira e Ballarin [15], que em estudos com madeiras maciças obtiveram coeficientes de determinação que variaram entre 0,76 e 0,89 . 


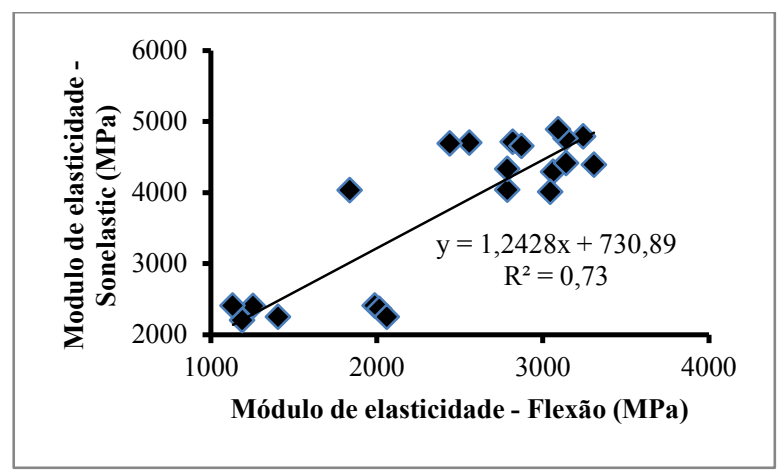

Figura 6: Gráfico da correlação entre os módulos de elasticidade

\section{CONCLUSÕES}

Com esse trabalho, pode se concluir que:

A umidade do compensado quando reutilizado têm pequeno aumento não comprometendo nas suas propriedades mecânicas. Já a densidade, devido a retirada de material ocasionada por pregos e ligação com concreto, ocasiona grande variação nas propriedades mecânicas do compensado.

Quanto maior o reuso do compensado de madeira, menor é o módulo de elasticidade, influenciando na estabilidade da forma de concreto, podendo prejudicar na geometria das peças de concreto.

O ensaio de excitação por impulso é eficiente para caracterização do compensado de madeira. A estimativa do módulo de elasticidade, da forma de concreto tanto sem uso como reutilizada, apresenta um coeficiente de correlação muito elevada, indicando ser um método eficaz.

No dimensionamento de formas de concreto, no estado limite de utilização, deve ser levado em consideração a diminuição do módulo de elasticidade.

\section{BIBLIOGRAFIA}

[1] NETTO, H. T. "Construção com madeira: um mercado promissor no Brasil”, Revista Exame, Abril 2016, disponível em: <http://exame.abril.com.br/negocios/dino/noticias/construcao-com-madeira-um-mercadopromissor-no-brasil.shtml>. Acesso em: 10 out. 2016.

[2] LANGENBACH, R., "Building Tall With Timber: A Pean to Wood Construction", Journal of the International Association for Bridge and Structural Engineering, v. 18, n. 2, pp.130-132, 2008.

[3] OLIVEIRA, J. T. S., FREITAS, A. R. "Painéis à base de madeira", Boletim Técnico do Departamento de Engenharia da Construção Civil - Escola Politécnica - Universidade de São Paulo. BT/PCC/149, EPUSP, n.188, São Paulo, 44p, 1995.

[4] STAMATO, G. C., Ligações em estruturas de madeira composta por chapas de madeira compensada, Tese de D.Sc., Escola de Engenharia de São Carlos, USP, São Paulo, 2002

[5] ANÔNIMO (2004), NBR 14931: Execução de estruturas de concreto - Procedimentos. Rio de Janeiro, ABNT - Associação Brasileira de Normas Técnicas.

[6] ARAÚJO, L. O. C., FREIRE, T. M. Tecnologia e gestão de sistemas construtivos de edifícios: Notas de aula da disciplina Tecnologia de Produção de Edificações em Concreto Armado. São Carlos: Departamento de Engenharia Civil, 2004, 86p.

[7] NBR 15696: Fôrmas e escoramentos para estruturas de concreto - Projeto, dimensionamento e procedimentos executivos. Rio de Janeiro, ABNT - Associação Brasileira de Normas Técnicas.

[8] OTANI, L.B., PEREIRA, A. H. A.. Guia de caracterização dos módulos elásticos e do amortecimento de madeiras e derivados utilisando as soluções SONELASTIC, Ribeirão Preto, ATCP Engenharia Física, 2013.

[9] ANÔNIMO (2011), NBR- 9485: Painéis de madeira compensada: determinação da massa específica. Rio de Janeiro, ABNT - Associação Brasileira de Normas Técnicas.

[10] ANÔNIMO (2011), NBR-9484: Painéis de madeira compensada: determinação do teor de umidade. Rio de Janeiro, ABNT - Associação Brasileira de Normas Técnicas.

[11] ANÔNIMO (2012), NBR- 9433: Painéis de madeira compensada: determinação dos módulos de elasticidade em resistência à flexão. Rio de Janeiro, ABNT - Associação Brasileira de Normas Técnicas. 
[12] ANÔNIMO (2006), E1876 - 09: Standard Test Method for Dynamic Young's Modulus, Shear Modulus, and Poisson's Ratio by Impulse Excitation of Vibration, New York, ASTM - American Society for Testing Materials.

[13] GARCIA, J. N., JUNIOR, G. B. "Propiedades de Resistência e Rigidez à flexão de painéis OSB e Compensados", Sociedade de Investigações Florestais, v.28, n.4, pp. 563-570, 2004.

[14] STANGERLIN, D. M., MELO, R. R., GATTO, D. A., et al., "Propriedades de Flexão estática da madeira de Carya illinoinensis em duas condições de umidade", Ciência da Madeira, v. 1, n. 2, pp. 70-79, 2010.

[15] NOGUEIRA, M., BALLARIN, A.W. "Correlação entre módulos de elasticidade estático (flexão) e dinâmico (ultra-som) para algumas espécies de eucalipto", In: Congresso Ibero-Americano de Pesquisa e Desenvolvimento DE PRODUTOS Florestais, Anais CD ROM, Curitiba, Fev. 2002. 